\title{
Endonasal endoscopic resection of olfactory neuroblastoma: an 11-year experience
}

\author{
Gary L. Gallia, MD, PhD, ${ }^{1-3}$ Anthony O. Asemota, MD, MPH, ${ }^{1}$ Ari M. Blitz, MD, ${ }^{4}$ \\ Andrew P. Lane, MD, ${ }^{2}$ Wayne Koch, MD, ${ }^{2}$ Douglas D. Reh, MD, ${ }^{1,2}$ and Masaru Ishii, MD, $\mathrm{PhD}^{1,2}$ \\ Departments of ${ }^{1}$ Neurosurgery, ${ }^{2}$ Otolaryngology/Head and Neck Surgery, and ${ }^{3}$ Oncology, and ${ }^{4}$ Division of Neuroradiology, \\ Department of Radiology and Radiological Science, Johns Hopkins University School of Medicine, Baltimore, Maryland
}

\begin{abstract}
OBJECTIVE Olfactory neuroblastoma (ONB) is a rare malignant neoplasm of the sinonasal cavity. Surgery has been and remains a mainstay of treatment for patients with this tumor. Open craniofacial resections have been the treatment of choice for many decades. More recently, experience has been growing with endoscopic approaches in the management of patients with ONB. The object of this study is to report the authors' experience over the past 11 years with ONB patients treated with purely endonasal endoscopic techniques.
\end{abstract}

METHODS The authors performed a retrospective chart review of 20 consecutive patients with ONB who underwent a completely endonasal endoscopic approach for an oncological tumor resection at their institution between January 2006 and January 2017. Patient demographics, tumor stage, pathological grade, frozen section analysis, permanent margin assessment, perioperative complications, postoperative therapy, length of follow-up, and outcomes at last follow-up were collected and analyzed.

RESULTS Eighteen patients presented with newly diagnosed disease, with a modified Kadish stage of A in 2 cases, B in $3, C$ in 11, and $D$ in 2. Two patients presented with recurrent tumors. An average of 25.3 specimens per patient were examined by frozen section analysis. Although analysis of intraoperative frozen section margins was negative in all but 1 case, microscopic foci of tumor were found in 7 cases (35\%) on permanent histopathological analysis. Perioperative complications occurred in 7 patients (35\%) including 1 patient who developed a cerebrospinal fluid leak; there were no episodes of meningitis. All but 1 patient received postoperative radiotherapy, and 5 patients received postoperative chemotherapy. With a mean follow-up of over 5 years, 19 patients were alive and 1 patient died from an unrelated cause. There were 2 cases of tumor recurrence. The 5 -year overall, disease-specific, and recurrence-free survival rates were $92.9 \%, 100 \%$, and $92.9 \%$, respectively.

CONCLUSIONS The current results provide additional evidence for the continued use of endoscopic procedures in the management of this malignancy.

https://thejns.org/doi/abs/10.3171/2018.2.JNS171424

KEYWORDS endoscopic; esthesioneuroblastoma; frozen section analysis; margin; olfactory neuroblastoma; oncology; outcomes; skull base surgery

$\mathrm{O}$ LFACTORY neuroblastoma (ONB), or esthesioneuroblastoma, is one of the neuroendocrine malignancies of the sinonasal tract. ${ }^{32}$ This entity was first described in $1924,{ }^{2}$ has a reported incidence of 0.4 cases per million, ${ }^{34}$ and accounts for approximately $6 \%$ of all sinonasal malignancies. ${ }^{35}$ These tumors are locally invasive and involve the nasal cavity, paranasal sinuses, and cribriform plate. There is often intracranial extension, and
ONBs can also invade the orbit. Neck disease occurs in approximately $20 \%$ of patients..$^{14}$

Given the rarity of ONB, there have been various treatment strategies. Surgery has been and remains a central component of treatment, with the majority of patients receiving surgery and radiotherapy.,7,17,25 From a surgical perspective, ONB has been traditionally approached with an open craniofacial resection (CFR). ${ }^{24}$ Over the past

ABBREVIATIONS CFR = craniofacial resection; DFS = disease-free survival; DSS = disease-specific survival; FDG = 2-deoxy-2-[18F]fluoro-D-glucose; IQR = interquartile range; $\mathrm{JHH}=$ Johns Hopkins Hospital; ONB = olfactory neuroblastoma; OS = overall survival; RFS = recurrence-free survival.

SUBMITTED July 11, 2017. ACCEPTED February 8, 2018.

INCLUDE WHEN CITING Published online August 3, 2018; DOI: 10.3171/2018.2.JNS171424. 
couple of decades, development of endoscopic techniques has provided additional surgical options for patients with sinonasal malignancies, including ONB. ${ }^{30}$ We previously reported our initial experience managing ONB with completely endoscopic techniques and margin assessment based on contiguous circumferential tissue analysis. ${ }^{13}$ In this report, we update this initial experience ${ }^{13}$ and present a series of 20 consecutive patients treated over the past 11 years at a single skull base center.

\section{Methods}

Under IRB-approved protocols, the Johns Hopkins Hospital (JHH) Pathology and Endoscopic Skull Base Center Databases were queried for patients who were treated surgically for ONB by the authors between January 2006 and January 2017 at JHH. Patients who underwent a completely endonasal endoscopic approach for an oncological tumor resection using the principles described in Gallia et al. ${ }^{13}$ were included. Patients who underwent an endoscopeassisted resection, where a craniotomy was combined with an endonasal endoscopic approach, or an open CFR were excluded. Patient demographics, tumor stage, pathological grade, frozen section analysis, permanent margin assessment, perioperative complications, postoperative therapy, length of follow-up, and outcomes at last follow-up were collected via retrospective chart review and analyzed. Patient consent was not required for this study. Modified Kadish staging was utilized: stage A, tumor confined to the nasal cavity; stage B, tumor confined to the nasal cavity and paranasal sinuses; stage $\mathrm{C}$, tumor extent beyond nasal cavity and paranasal sinuses, including involvement of the cribriform plate, base of the skull, orbit, or intracranial cavity; stage D, tumor with metastasis to cervical lymph nodes or distant sites. ${ }^{22}$ Histopathological grading was according to Hyams. ${ }^{15}$

We have previously described our preoperative evaluation, surgical approach, and postoperative management for patients with sinonasal malignancies including ONB..$^{13,30}$ Preoperative high-resolution skull base imaging is performed with both CT and MRI to determine the extent of disease and resectability via a purely endoscopic approach. ${ }^{3,28}$ A preoperative PET/CT scan with 2-deoxy$2-\left[{ }^{18} \mathrm{~F}\right]$ fluoro-D-glucose (FDG) is currently standard in our practice to evaluate for systemic disease. Patients are considered for an expanded endonasal endoscopic approach should the extent of tumor, including tissue for margin assessment, be accessible via an endonasal surgical corridor. Circumferential contiguous margins are analyzed from the sinonasal cavity/septum, extracranial skull base, and intracranial space including the dura, falx, and olfactory nerves..$^{13,30}$ There are various skull base reconstruction methods, including nasal vascularized flaps, acellular dermal grafts, and synthetic materials. ${ }^{12,13}$

Statistical analyses employed standard descriptive techniques to characterize the study population. Overall survival (OS) was determined from the date of resection to death from any cause or last follow-up. Disease-specific survival (DSS) was determined from the date of resection to death directly attributable to ONB or last follow-up. Recurrence-free survival (RFS) was determined from the date of surgery to date of recurrent ONB or last followup. For OS and DSS analyses, last follow-up was defined as the most recent communication with a JHH physician. For recurrence analysis, last follow-up was defined as the most recent evaluation of disease status by a member of the patient's oncology team (neurosurgeon, otolaryngologist, radiation oncologist, and/or medical oncologist) at which a disease assessment was performed. For patients undergoing a staged resection, OS, DSS, and RFS were calculated from the date of the final surgical procedure. Five-year OS, DSS, and RFS, reported as percentages, were obtained from the survivor function, which was estimated by the Kaplan-Meier method. ${ }^{19}$ For patients who presented to JHH with recurrent disease, time zero for survival analyses was defined as the date of resection of the recurrent tumor.

\section{Results}

A total of 28 patients were identified. Twenty of these patients underwent a purely endoscopic approach for an oncological resection of an ONB, and their data were analyzed (Table 1). Four patients who underwent an endoscope-assisted resection (1 patient with stage B, 3 patients with stage C), 3 patients who underwent an open CFR (all stage $\mathrm{C}$ ), and 1 patient who underwent a parotidectomy and neck dissection were excluded from analysis. The mean age of the 20 included patients was 51 years (range 34-73 years), and the male to female ratio was 1.9:1. Nasal congestion and sinusitis were the most common presenting symptoms. Eighteen patients (90\%) presented with newly diagnosed disease, and $2(10 \%)$ presented with recurrent tumors. Prior to definitive resection, all patients underwent an endonasal endoscopic biopsy and/or partial resection and specimens were reviewed by the JHH Pathology Department and interpreted as ONB. Of the patients with newly diagnosed disease, 11 presented with stage $C, 3$ with stage $\mathrm{B}, 2$ with stage $\mathrm{A}$, and 2 with stage $\mathrm{D}$. Both patients with recurrent tumors had prior surgery as a sole treatment modality -17 months prior to presentation at $\mathrm{JHH}$ in one case and 61 months prior to presentation at JHH in the other. At recurrence, one of these patients had stage B disease and the other stage $\mathrm{C}$ disease. The Hyams grade was 1 in 3 cases (15\%), 2 in 11 cases (55\%), and 3 in 6 cases (30\%). Seventeen patients, and all patients treated since 2010, had preoperative PET/CT scans. Three of these patients, all with newly diagnosed disease, had cervical FDG activity.

All patients underwent a purely endonasal endoscopic approach, performed with the intention of obtaining negative intraoperative circumferentially contiguous margins. Fifteen patients $(75 \%)$ underwent a single surgical procedure, and 5 patients $(25 \%)$ underwent staged procedures. In patients with FDG neck activity, surgery was performed in a staged fashion, with the first stage comprising a neck dissection and resection of the sinonasal component of tumor with intraoperative margin assessment; stage 2 consisted of resection of the skull base, intraoperative margin assessment, and skull base reconstruction. In 2 other patients, cases were staged due to the length of the procedure. Four patients had a unilateral resection.

An average of 25.3 frozen specimens (median 26, range 
4-41) were analyzed per patient. In all but 1 patient, negative intraoperative margins were achieved on frozen histopathological analysis. In 1 patient, mucosa in the anterolateral aspect of the frontal sinus contained atypical nests suspicious for tumor; there was no remaining mucosa in this region to obtain a superseding soft tissue specimen. In 7 patients (35\%), at least 1 margin that was negative on frozen analysis had microscopic foci of tumor on permanent analysis. Of these specimens (10 in total), 5 were obtained from mucosa in the sinonasal cavity/septum, 4 were from the extracranial skull base, and 1 specimen was from the intracranial space (dura). Two of these patients were taken back to the operating room for re-resection of the involved margin. In both cases, all specimens from the re-resection were negative on frozen and permanent analyses. In the remaining 5 cases, no additional surgery was performed for one of several reasons: 1) additional tissue had been removed well beyond the intraoperative false negative specimen during the initial surgery (2 patients), 2) patients were planning on proceeding with chemotherapy ( 2 patients; 1 with stage $\mathrm{D}$ and the patient with the positive margin), and 3 ) the patient declined further surgery (1 patient). Two of the 3 patients undergoing a neck dissection had cervical metastatic disease.

Perioperative complications occurred in 7 patients (35\%). One patient developed an intraoperative hypertensive crisis due to a catecholamine-secreting ONB.$^{31}$ Three patients developed postoperative surgical complications. Two of these 3 patients had more than expected pneumocephalus, and in both cases it was managed conservatively by early removal of the lumbar drain. The third patient had a skull base CSF leak that required additional surgery; this patient was also one of the 2 who underwent re-resection of a margin that was negative on intraoperative frozen section analysis during the initial resection but positive for tumor on permanent section. Three patients experienced postoperative medical complications. One patient with a history of atrial fibrillation developed a pulmonary embolism, 1 patient had Clostridium difficile colitis, and 1 patient developed transient atrial fibrillation.

Nineteen patients $(95 \%)$ received postoperative radiotherapy, and 5 (25\%) received postoperative chemotherapy. Indications for chemotherapy included stage $\mathrm{D}$ disease in 2 patients, stage $\mathrm{C}$ disease and extensive mucosal spread in 2 patients (including the patient with the positive margin), and stage B disease with unilateral resection of a high grade (Hyams 3) tumor in 1 patient.

The overall mean duration of follow-up was 71.3 months (range 16-141 months), the median follow-up time was 70.4 months (interquartile range [IQR] 46.1-95.8 months), and the mean time between the last reported follow-up and manuscript submission was 4.2 months (range 0-10 months). There were no patients lost to follow-up. Twelve patients have been followed for at least 5 years since treatment at JHH. Nineteen patients (95\%) were alive at last follow-up. One patient who presented with newly diagnosed disease died from an unrelated cause (pancreatic cancer) 56 months after surgery and had no evidence of recurrent ONB at the time of death. The 5-year OS and DSS were $92.9 \%$ (95\% CI 59.1\%-99\%) and 100\%, respectively (Figs. 1 and 2). For recurrence analysis, the mean onco-
TABLE 1. Characteristics of study cohort

\begin{tabular}{|c|c|}
\hline Characteristic & Value \\
\hline New diagnosis & $18(90.0)$ \\
\hline Recurrent cases & $2(10.0)$ \\
\hline Mean age at diagnosis (yrs) & $51.1 \pm 12.2$ \\
\hline Male sex & $13(65.0)$ \\
\hline \multicolumn{2}{|l|}{ Symptoms } \\
\hline Congestion & $11(55.0)$ \\
\hline Sinusitis & $10(50.0)$ \\
\hline Anosmia/hyposmia/dysosmia & $9(45.0)$ \\
\hline Obstruction & $8(40.0)$ \\
\hline Epistaxis & $6(30.0)$ \\
\hline Discharge & $5(25.0)$ \\
\hline Headache & $4(20.0)$ \\
\hline Facial pain & $3(15.0)$ \\
\hline Visual changes & $2(10.0)$ \\
\hline Cough & $2(10.0)$ \\
\hline Nasal mass & $2(10.0)$ \\
\hline \multicolumn{2}{|l|}{ Kadish staging } \\
\hline A & $2(10.0)$ \\
\hline$B$ & $4(20.0)$ \\
\hline C & $12(60.0)$ \\
\hline $\mathrm{D}$ & $2(10.0)$ \\
\hline \multicolumn{2}{|l|}{ Hyams grade } \\
\hline 1 & $3(15.0)$ \\
\hline 2 & $11(55.0)$ \\
\hline 3 & $6(30.0)$ \\
\hline \multicolumn{2}{|l|}{ Preop evaluation } \\
\hline Preop PET/CT & $17(85.0)$ \\
\hline Cervical FDG activity & $3(15.0)$ \\
\hline
\end{tabular}

Values are number of cases (\%) unless otherwise indicated. Mean age is presented with SD.

logical follow-up was 65 months (range 16-141 months), the median oncological follow-up was 57.5 months (IQR 33.3-93.6 months), and the mean time between last oncological follow-up and manuscript submission was 11.3 months (range 0-60 months). Ten patients have had more than 5 years of oncological follow-up. Two patients (10\%) experienced recurrent $\mathrm{ONB}$, including 1 patient who initially developed a local recurrence and 1 who developed neck metastasis. One patient with newly diagnosed stage $\mathrm{C}$ ONB treated with radiotherapy following a bilateral resection developed an intraorbital recurrence 43 months after surgery. This recurrence was treated with re-irradiation. Eighteen months later, the patient developed neck disease and underwent bilateral neck dissections and additional radiotherapy. The patient subsequently developed small subdural deposits, which were treated with radiosurgery, and has been in clinical trials for progressive orbital and intracranial disease. This patient is currently 114 months out from initial surgery, off all treatment, with stable disease. The second patient who experienced ONB recurrence was also initially treated for newly diagnosed stage $\mathrm{C}$ disease; 


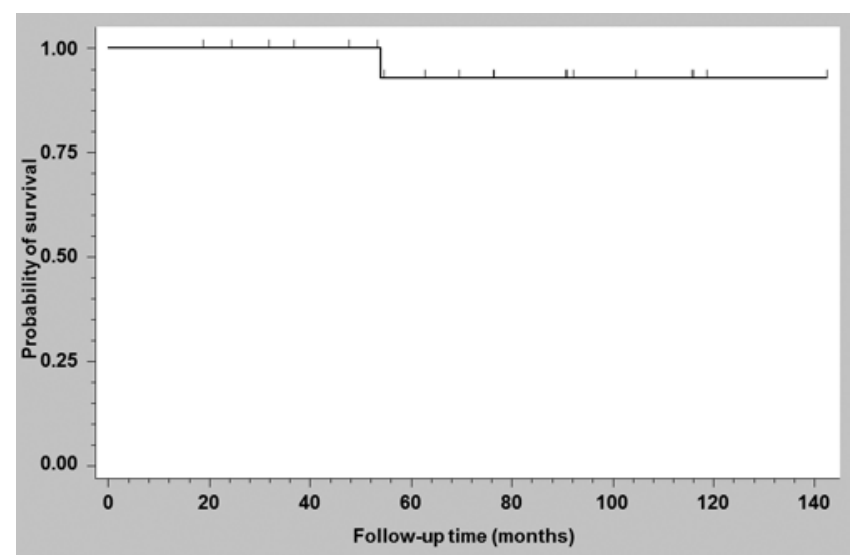

FIG. 1. Kaplan-Meier estimate of overall survival (OS). Mean follow-up time was 71.3 months. The 5 -year OS was $92.9 \%$. Death was from pancreatic cancer unrelated to primary disease.

the patient's preoperative PET/CT scan was negative for systemic disease. After endoscopic bilateral resection, this patient was treated with postoperative chemoradiotherapy for extensive mucosal spread. Sixty-one months after resection, the patient was found to have FDG activity in the soft tissue anterior to the clivus and in the right neck. An endoscopic approach was performed for resection of the FDG-avid nasopharyngeal/clival tissue and neck dissection. The nasopharyngeal/clival pathology was negative for tumor; neck pathology confirmed metastatic ONB. The patient subsequently underwent bilateral neck irradiation and is now 52 months out from his last surgery with no evidence of disease. The 5-year RFS was 92.9\% (95\% CI $59.1 \%-99 \%$ ) (Fig. 3).

\section{Discussion}

Central to contemporary surgical approaches in patients with sinonasal malignancies is the ability to obtain a negative margin resection; other key surgical principles include preservation of critical neurovascular structures, separation of intracranial and sinonasal compartments, and optimizing cosmesis. Given the origin of ONB in the superior aspect of the nasal cavity, in the majority of cases, obtaining a negative margin necessitates resection of the cribriform plate, dura, and olfactory bulbs/distal nerves. Surgical approaches to achieve these goals and in particular an oncological resection have traditionally included an open CFR. The largest study of open craniofacial surgery in ONB patients was reported by an international collaborative study group and included 151 patients from 17 investigators up to $2000 .{ }^{24}$ In this study, which included 33 patients with Kadish stage A/B disease and 116 with Kadish stage $\mathrm{C}$ disease, the 5-year OS, DSS, and RFS rates were $78 \%, 83 \%$, and $64 \%$, respectively. Overall, $32 \%$ of patients developed a complication; central nervous system and wound complications were the most common. ${ }^{24}$

Over the past couple of decades, there has been tremendous development and application of endoscopic approaches to skull base pathologies, including sinonasal malignancies. ${ }^{30}$ With respect to ONB, the earliest reports of a purely endoscopic approach were in the late 1990s. ${ }^{1,33}$

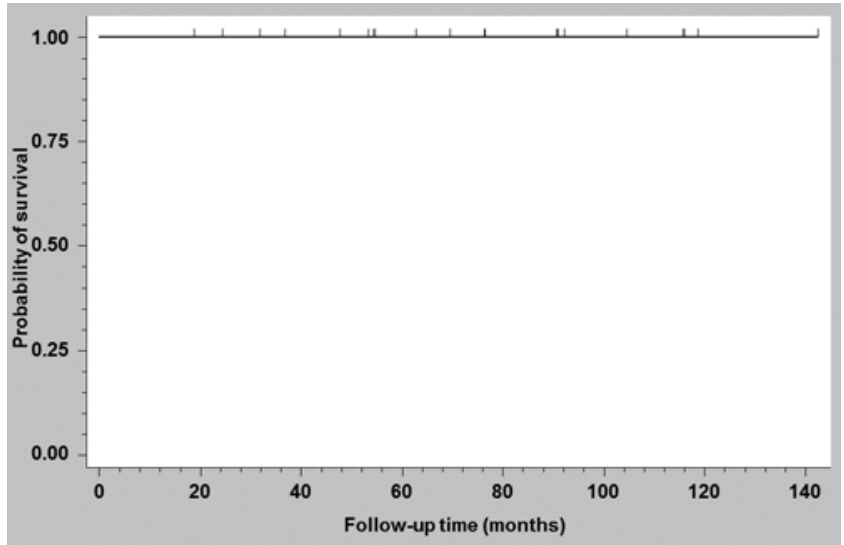

FIG. 2. Kaplan-Meier estimate of disease-specific survival (DSS). The mean follow-up time was 71.3 months. The 5 -year DSS was $100 \%$.

Since that time, there have been numerous case reports and series of ONB patients treated with completely endoscopic approaches. ${ }^{29}$ It is important to note that the surgical principles of skull base surgery remain unchanged in the application of more recent techniques and approaching a particular case endoscopically should not compromise these principles. Certainly, in some cases, a negative margin resection cannot be achieved, irrespective of the approach used, but the main driving principle in consideration of surgical techniques should remain focused on the approach that can result in the best oncological outcome.

It is not surprising, given the rarity of $\mathrm{ONB}$, that the majority of reports detailing management of ONB with purely endoscopic techniques contain small number of patients. In fact, there are only a few studies that include more than 20 patients treated solely endoscopically. In a study reported by Folbe et al. ${ }^{10}$ data from 23 patients with ONB treated endoscopically at 2 centers were analyzed. In that study, 19 patients were treated for primary disease and 4 for recurrence. For the 19 patients with initial disease, modified Kadish staging was A in 2 patients (10.5\%), B in 11 patients $(58.9 \%), \mathrm{C}$ in 5 patients $(26.3 \%)$, and $\mathrm{D}$ in 1 patient $(5.3 \%)$. Complete resection and negative intraoperative margins were reported in 17 of the patients undergoing primary treatment. One patient required a craniotomy for complete resection of a positive lateral dural margin, and another patient had positive margins at the orbital apex. Four CSF leaks were reported. Sixteen patients (69.6\%) had postoperative radiotherapy. The mean duration of follow-up was 45.2 months. All patients with primary disease were without evidence of disease at last follow-up. Of the 4 patients with recurrent disease at presentation, 3 were without disease at last follow-up. ${ }^{10}$

In a study by Feng et al., ${ }^{9} 24$ ONB patients were treated endoscopically. In that study, 19 patients presented with newly diagnosed ONB and 5 with recurrence. Both Kadish $^{18}$ and Dulguerov ${ }^{8}$ classifications were reported. Three (12.5\%), 6 (25\%), and 15 (62.5\%) patients had Kadish stage A, B, and C, respectively; 9 (37.5\%), 7 (29.2\%), 2 $(8.3 \%)$, and $6(25 \%)$ patients had T1, T2, T3, and T4, respectively. Complete resection and negative margins were reported in 19 patients (79.2\%). Interestingly, although in the majority of patients (62.5\%), tumor mainly involved the 


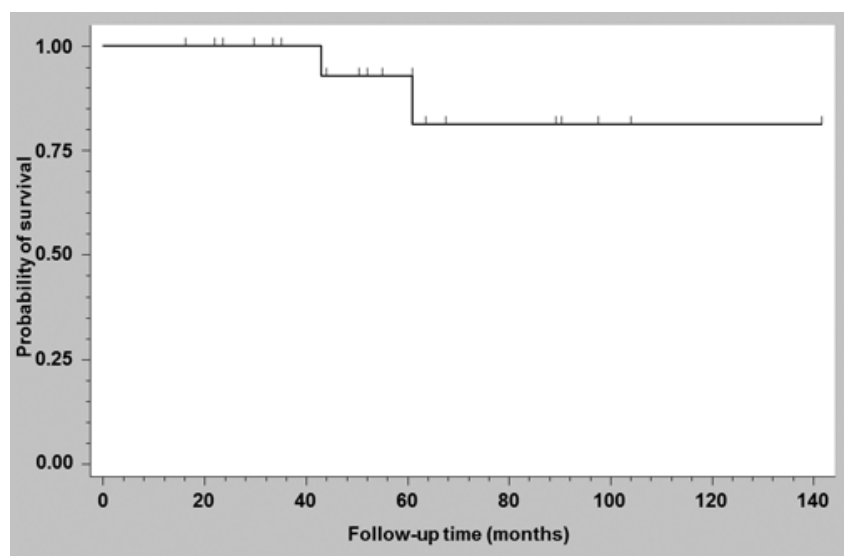

FIG. 3. Kaplan-Meier estimate of recurrence-free survival (RFS). The mean oncological follow-up time was 65 months. The 5-year RFS was $92.9 \%$.

cribriform plate and skull base, only 4 patients were reported to have resection of the entire cribriform plate, dura, and olfactory bulb. Twenty patients $(83.3 \%)$ received postoperative radiotherapy and 7 patients $(29.2 \%)$ also received chemotherapy. One patient developed a CSF leak. The median duration of follow-up was 44 months. Four (16.6\%) patients, all with Kadish stage C disease, died of tumor recurrence; the 3-year OS and disease-free survival (DFS) rates were $82 \%$ and $70.8 \%$, respectively. ${ }^{9}$ These results are lower than in our current study and in other groups' experiences. ${ }^{10,27}$ We agree with the authors 9 that the involvement of the cribriform is an important prognostic factor in recurrence when extradural resections are performed and we advocate for initial resection of the cribriform plate, dura, and olfactory bulbs/nerves with contiguous circumferential margin assessment when the cribriform is involved.

In a recent study by Lund and $\mathrm{Wei}^{21}$ on endoscopic surgery for sinonasal malignancies, the authors reported 140 cases between 1996 and 2014 including 36 with ONB. Postoperative radiotherapy was given to 33 ONB patients (91.7\%) and combined with chemotherapy in 25 patients (69.4\%). Mean follow-up in this study for patients with ONB was 77.6 months (range 6-162 months). The authors reported a $97 \%$ OS at both 5 and 10 years and a 90\% DFS at both time points. Mean OS and DFS were 162 and 150 months, respectively. ${ }^{21}$ In a separate study, these authors compared endoscopic and open surgical techniques in patients with ONB ${ }^{27}$ In this 35 -year experience with 95 patients who underwent surgery for ONB between 1978 and 2013, 65 patients underwent a CFR and 30 underwent a purely endoscopic approach. The majority of patients received postoperative radiotherapy and after 2003 all patients received postoperative chemoradiotherapy, unless contraindicated. Mean follow-up was 90 months (range 1-309 months) in patients who underwent a CFR and 73 months (range 3-143 months) in patients undergoing an endoscopic resection. OS and DFS were significantly improved in the endoscopic group compared to the CFR cohort. The 5- and 10-year OS in the CFR group were 76.5\% and $67.8 \%$, respectively and in the endoscopic group these values were $100 \%$. The 5- and 10-year DFS were $73.5 \%$ and $57.1 \%$, respectively in the CFR group and $96 \%$ and
$75.6 \%$ in the endoscopic group. Patients with more advanced disease were significantly more likely to undergo a craniofacial compared to endoscopic resection and patients treated endoscopically were significantly more likely to receive postoperative chemoradiotherapy. ${ }^{27}$ These studies highlight another important question in the management of patients with ONB, that is, the role of chemotherapy. Given the rarity of this disease, it is not surprising that there are various treatment algorithms. In our practice, chemotherapy is added in select situations and in our current endoscopic cohort, only $25 \%$ of patients received chemotherapy following surgery.

In addition to the above and other studies on $\mathrm{ONB},{ }^{29}$ there are a few meta-analysis and systematic reviews of the literature comparing various surgical approaches in the management of this malignancy. In a meta-analysis of 23 studies between 1992 and 2008 comprising 361 patients reported by Devaiah and Andreoli, ${ }^{5}$ better survival rates were found in patients treated with endoscopic surgery compared to open surgery. This comparative analysis is confounded by the fact that most patients who underwent open surgery were Kadish stages C and D, whereas the patients undergoing endoscopic techniques more commonly were Kadish stages A and B; given the limited numbers, stratification by stage was not possible. ${ }^{5}$

In a systematic literature review, Komotar et al. compared outcomes between patients undergoing a CFR, a cranionasal (endoscope-assisted) approach, and a purely endoscopic endonasal approach. ${ }^{20}$ A total of 47 studies published between 1985 and 2010 containing 453 patients were included in this analysis. In this report, rates of local recurrence were lower and rates of negative margins were higher among patients undergoing purely endoscopic procedures (8\% and $93.8 \%$, respectively) and endoscopeassisted procedures $(16.7 \%$ and $95.8 \%$, respectively) than among patients undergoing a CFR $(22.1 \%$ and $77.3 \%$, respectively). ${ }^{20}$ Similar to the Devaiah and Andreoli study, ${ }^{5}$ there were a greater proportion of lower-grade tumors and fewer higher-grade tumors in the endoscopic group compared with the craniofacial group and, consequently, direct comparisons of outcomes, complications, and survival were not possible between groups. ${ }^{20}$

In a recent systematic review and meta-analysis, $\mathrm{Fu}$ et al. evaluated 36 studies containing 609 patients comparing outcomes for open versus endoscopic resection of ONB..$^{11}$ In this analysis, endoscopic surgery was defined as a purely endoscopic approach; open surgery included transcranial, transfacial, and endoscope-assisted procedures. In the aggregate participant data analysis, patients undergoing an endoscopic resection had significantly lower rates of locoregional recurrence (17.4\% versus $45 \%$ ), distant metastasis $(1.1 \%$ versus $7.5 \%)$, cause-specific mortality ( $0 \%$ versus $15.2 \%$ ), and overall mortality (0\% versus $19.9 \%$ ) compared to open surgery. In the pooled individual participant data analysis, endoscopic surgery was associated with significantly higher rates of OS and DFS on univariate analysis. This was not significant, however, on multivariate analysis when controlled for age, stage, chemotherapy, and radiotherapy. In a subgroup analysis, the authors reported that patients with Kadish stage C/D had improved OS and a trend to improvement in DSS when treated endoscopically 
compared to open surgery. Patients with advanced Hyams grade (III/IV) also had significantly higher OS and DSS when treated endoscopically compared to open surgery.11

As noted previously, a central tenet to skull base surgery for sinonasal malignancies is the goal of achieving a negative margin resection. Not surprisingly, given the rarity of ONB, there are only a handful of studies which report on margin status in ONB. In the largest of these studies, Patel et al. found positive (including close) surgical margins predictors of worse OS ( $p=0.02)$, DSS ( $p=0.04)$, and RFS $(p=0.01)$. In a study by Van Gompel et al., circumferential negative margins were found associated with improved survival. ${ }^{36}$ In the study by Resto et al., the most significant predictor of decreased time to recurrence and decreased OS was positive surgical margins. ${ }^{26}$ There are other studies, however, that did not find these associations, ${ }^{6}$ and notably the majority of studies do not analyze surgical margins.

This topic is likely more complex than on cursory review. For example, are the reported surgical margins specimen margins or resection-cavity margins? Are these contiguous circumferential margins or are they from selected regions of the specimen or resection cavity? Is the intraoperative frozen section analysis or permanent histopathological findings most important (i.e., what is the impact of a microscopic focus of tumor on permanent histopathological analysis)? Moreover, is there a difference in RFS, DSS, and/or OS between these variables? In our practice, we obtain circumferential contiguous tissue around the tumor for frozen section analysis. ${ }^{13,30}$ For the sinonasal and extracranial margins, these are obtained with the tumor in situ (often debulked) and are best considered resection cavity margins. For the cribriform/dural margins, these are most often specimen margins; however, resection cavity margins have also been analyzed. Irrespective of the margin location, tissue is obtained in a contiguous fashion circumferentially around the tumor.

In our cohort, we achieved negative intraoperative margins on frozen section analysis in all but 1 case. On permanent histopathological analysis, however, we found microscopic foci of tumor cells in 35\% of cases. In a recently published study, ${ }^{16}$ we examined the performance of frozen section analysis in ONB and found a sensitivity of 0.89 , a specificity of 0.96 , an accuracy of 0.95 , a positive predictive value of 0.86 , a negative predictive value of 0.97 , a likelihood ratio positive of 24.38 , a likelihood ratio negative of 0.11 , and a prevalence of 0.20 . Based on these metrics and the number of samples we take per case, which in this study averaged 25.3, the finding of falsely negative frozen margins in 35\% of cases is not surprising. It is somewhat unclear what to do in such cases, as often there is more tissue removed external to the falsely negative intraoperative specimen, most patients receive adjuvant radiotherapy, some patients will receive chemotherapy, and there is often a long time to recurrence. In our practice, we perform a risk assessment of the probability of residual tumor by reviewing the specimens with our head and neck pathology colleagues and intraoperative documentation, including photographs and video; discuss the various options with the patient; and present these cases at our multidisciplinary head and neck tumor board. In cases where the risk is low, such as in a case where there is a microscopic focus of cells and tissue was removed distal to this region or in patients in whom chemotherapy is planned, we typically proceed with adjuvant radiotherapy or chemoradiotherapy, respectively. In cases in which we feel the risk of disease is moderate to high, we recommend returning to the operating room for re-resection. In 2 cases, we did just this, and in both cases, the results of analysis of the frozen and permanent specimens obtained at re-resection were negative. Additional studies reporting outcomes in similar situations will be needed to further clarify the optimal management strategy in such cases.

There are several limitations of our study, including the retrospective design. In addition, although this is one of the largest single-center studies reporting outcomes for ONB patients treated purely endoscopically, this study only contains a cohort of 20 patients. Moreover, ONB recurrence often occurs in a delayed fashion. In the systematic review by Komotar et al., ${ }^{20}$ the average time to local recurrence in patients who underwent purely endoscopic resection of ONB was 33 months, and recurrences can occur well past this time..$^{23,27}$ Thus, although our mean follow-up was over 5 years, continued surveillance is essential to determine long-term outcomes for ONB patients treated purely endoscopically.

\section{Conclusions}

This case series provides additional evidence for the continued use of endoscopic procedures in the oncological management of well-selected cases of ONB. Due to the delayed recurrences seen in ONB, long-term follow-up of larger patient cohorts is required to define the role of purely endoscopic approaches in the treatment of this malignancy.

\section{References}

1. Anderhuber W, Stammberger H, Walch C, Fock C, Regauer $\mathrm{S}$, Luxenberger $\mathrm{W}$, et al: Rigid endoscopy in minimally invasive therapy of tumors of the paranasal sinuses and skull base. Minim Invasive Ther Allied Technol 8:25-32, 1999

2. Berger L, Luc R, Richard D: L'esthesioneuroepitheliome olfactif. Bull Assoc Fr Etud Cancer 13:410-421, 1924

3. Blitz AM, Aygun N, Herzka DA, Ishii M, Gallia GL: High resolution three-dimensional MR imaging of the skull base: compartments, boundaries, and critical structures. Radiol Clin North Am 55:17-30, 2017

4. Broich G, Pagliari A, Ottaviani F: Esthesioneuroblastoma: a general review of the cases published since the discovery of the tumour in 1924. Anticancer Res 17 (4A):2683-2706, 1997

5. Devaiah AK, Andreoli MT: Treatment of esthesioneuroblastoma: a 16-year meta-analysis of 361 patients. Laryngoscope 119:1412-1416, 2009

6. Diaz EM Jr, Johnigan RH III, Pero C, El-Naggar AK, Roberts DB, Barker JL, et al: Olfactory neuroblastoma: the 22year experience at one comprehensive cancer center. Head Neck 27:138-149, 2005

7. Dulguerov P, Allal AS, Calcaterra TC: Esthesioneuroblastoma: a meta-analysis and review. Lancet Oncol 2:683-690, 2001

8. Dulguerov P, Calcaterra T: Esthesioneuroblastoma: the UCLA experience 1970-1990. Laryngoscope 102:843-849, 1992

9. Feng L, Fang J, Zhang L, Li H, Zhou B, Chen X, et al: Endoscopic endonasal resection of esthesioneuroblastoma: a single 
center experience of 24 patients. Clin Neurol Neurosurg 138:94-98, 2015

10. Folbe A, Herzallah I, Duvvuri U, Bublik M, Sargi Z, Snyderman $\mathrm{CH}$, et al: Endoscopic endonasal resection of esthesioneuroblastoma: a multicenter study. Am J Rhinol Allergy 23:91-94, 2009

11. Fu TS, Monteiro E, Muhanna N, Goldstein DP, de Almeida JR: Comparison of outcomes for open versus endoscopic approaches for olfactory neuroblastoma: A systematic review and individual participant data meta-analysis. Head Neck 38 (Suppl 1):E2306-E2316, 2016

12. Gallia GL, Reh DD, Lane AP, Higgins TS, Koch W, Ishii M: Endoscopic resection of esthesioneuroblastoma. J Clin Neurosci 19:1478-1482, 2012

13. Gallia GL, Reh DD, Salmasi V, Blitz AM, Koch W, Ishii M: Endonasal endoscopic resection of esthesioneuroblastoma: the Johns Hopkins Hospital experience and review of the literature. Neurosurg Rev 34:465-475, 2011

14. Gore MR, Zanation AM: Salvage treatment of late neck metastasis in esthesioneuroblastoma: a meta-analysis. Arch Otolaryngol Head Neck Surg 135:1030-1034, 2009

15. Hyams VJ: Olfactory neuroblastoma (case 6), in Batsakis JG, Hyams VJ, Morales AR (eds): Special Tumors of the Head and Neck. Chicago: ASCP Press, 1983, pp 24-29

16. Ishii M, Bishop JA, Gallia GL: Assessment of frozen section margin analysis during olfactory neuroblastoma surgery. Laryngoscope 127:1735-1741, 2017

17. Jethanamest D, Morris LG, Sikora AG, Kutler DI: Esthesioneuroblastoma: a population-based analysis of survival and prognostic factors. Arch Otolaryngol Head Neck Surg 133:276-280, 2007

18. Kadish S, Goodman M, Wang CC: Olfactory neuroblastoma. A clinical analysis of 17 cases. Cancer 37:1571-1576, 1976

19. Kaplan EL, Meier P: Nonparametric-estimation from incomplete observations. J Am Stat Assoc 53:457-481, 1958

20. Komotar RJ, Starke RM, Raper DM, Anand VK, Schwartz TH: Endoscopic endonasal compared with anterior craniofacial and combined cranionasal resection of esthesioneuroblastomas. World Neurosurg 80:148-159, 2013

21. Lund VJ, Wei WI: Endoscopic surgery for malignant sinonasal tumours: an eighteen year experience. Rhinology 53:204-211, 2015

22. Morita A, Ebersold MJ, Olsen KD, Foote RL, Lewis JE, Quast LM: Esthesioneuroblastoma: prognosis and management. Neurosurgery 32:706-715, 1993

23. Ow TJ, Hanna EY, Roberts DB, Levine NB, El-Naggar AK, Rosenthal DI, et al: Optimization of long-term outcomes for patients with esthesioneuroblastoma. Head Neck 36:524530, 2014

24. Patel SG, Singh B, Stambuk HE, Carlson D, Bridger PG, Cantu G, et al: Craniofacial surgery for esthesioneuroblastoma: report of an international collaborative study. J Neurol Surg B Skull Base 73:208-220, 2012

25. Platek ME, Merzianu M, Mashtare TL, Popat SR, Rigual NR, Warren GW, et al: Improved survival following surgery and radiation therapy for olfactory neuroblastoma: analysis of the SEER database. Radiat Oncol 6:41, 2011

26. Resto VA, Eisele DW, Forastiere A, Zahurak M, Lee DJ, Westra WH: Esthesioneuroblastoma: the Johns Hopkins experience. Head Neck 22:550-558, 2000
27. Rimmer J, Lund VJ, Beale T, Wei WI, Howard D: Olfactory neuroblastoma: a 35-year experience and suggested follow-up protocol. Laryngoscope 124:1542-1549, 2014

28. Roxbury CR, Ishii M, Blitz AM, Reh DD, Gallia GL: Expanded endonasal endoscopic approaches to the skull base for the radiologist. Radiol Clin North Am 55:1-16, 2017

29. Roxbury CR, Ishii M, Gallia GL, Reh DD: Endoscopic management of esthesioneuroblastoma. Otolaryngol Clin North Am 49:153-165, 2016

30. Roxbury CR, Ishii M, Richmon JD, Blitz AM, Reh DD, Gallia GL: Endonasal endoscopic surgery in the management of sinonasal and anterior skull base malignancies. Head Neck Pathol 10:13-22, 2016

31. Salmasi V, Schiavi A, Binder ZA, Ruzevick J, Orr BA, Burger PC, et al: Intraoperative hypertensive crisis due to a catecholamine-secreting esthesioneuroblastoma. Head Neck 37:E74-E80, 2015

32. Shah K, Perez-Ordóñez B: Neuroendocrine neoplasms of the sinonasal tract: neuroendocrine carcinomas and olfactory neuroblastoma. Head Neck Pathol 10:85-94, 2016

33. Stammberger H, Anderhuber W, Walch C, Papaefthymiou G: Possibilities and limitations of endoscopic management of nasal and paranasal sinus malignancies. Acta Otorhinolaryngol Belg 53:199-205, 1999

34. Theilgaard SA, Buchwald C, Ingeholm P, Kornum Larsen S, Eriksen JG, Sand Hansen H: Esthesioneuroblastoma: a Danish demographic study of 40 patients registered between 1978 and 2000. Acta Otolaryngol 123:433-439, 2003

35. Turner JH, Reh DD: Incidence and survival in patients with sinonasal cancer: a historical analysis of population-based data. Head Neck 34:877-885, 2012

36. Van Gompel JJ, Giannini C, Olsen KD, Moore E, Piccirilli M, Foote RL, et al: Long-term outcome of esthesioneuroblastoma: Hyams grade predicts patient survival. J Neurol Surg B Skull Base 73:331-336, 2012

\section{Disclosures}

The authors report no conflict of interest concerning the materials or methods used in this study or the findings specified in this paper.

\section{Author Contributions}

Conception and design: Gallia. Acquisition of data: Gallia, Blitz, Lane, Koch, Reh, Ishii. Analysis and interpretation of data: Gallia, Asemota, Blitz, Lane, Reh, Ishii. Drafting the article: Gallia. Critically revising the article: Gallia, Asemota, Ishii. Reviewed submitted version of manuscript: all authors. Approved the final version of the manuscript on behalf of all authors: Gallia. Statistical analysis: Asemota. Administrative/technical/material support: Gallia. Study supervision: Gallia.

\section{Correspondence}

Gary L. Gallia: Johns Hopkins University School of Medicine, Baltimore, MD.ggallia1@jhmi.edu. 